\title{
Affective Mapping of Social Networks
}

\author{
Monica Wachowicz \\ Wageningen UR \\ The Netherlands \\ monica.wachowicz@wur.nl
}

\author{
Teresa Iturrioz, Jorge Cano \\ Technical University of Madrid \\ Spain \\ teresa.iturrioz@upm.es \\ jcanofuentes@gmail.com
}

\author{
Amelia Polónia, Sara Pinto \\ University of Porto \\ Portugal \\ amelia.polonia@netc.pt \\ saramcpinto@gmail.com
}

\begin{abstract}
Very little is know about how the patterns of collective experiences towards a Place can quantitatively as well as qualitatively be represented based on subjective data. Therefore, our research challenge is two-fold: (a) to define what Place is by pointing to ways in which emotion states can be observed and are bound together within a social network; and (b) to develop a visual representation that provides the framework for representing patterns of emotion states associated to this Place. Towards this end, this paper will demonstrate our first results on the analysis of the correspondence obtained in the archive of Simón Ruiz that adds up to more than 50,000 documents, which were collected from a long list of sources during four decades.
\end{abstract}

\section{Introduction}

Affective mapping is about the visual representation of Place. A Place only exists when a space acquires its meaning with the presence of people and their human emotions towards this particular space at some particular period in time [1]. As a consequence of a personal experience of a space, we can witness the multiplication of Places generated from the collective and individual emotional states associated with a variety of feelings towards a space. The quiet, the hectic, the dangerous Places or simply those Places that make us feel happy can surely be measured, analysed and structured through a visual representation. In the past, psycho-geography has tried to understand the role of a Place in shaping our emotions; but now affective mapping is trying to put together subjective experiences and personal tales in order to gradually determine patterns of emotions that lead to a visual representation of a Place.

\section{Social Networks}

One interesting example we first came across was the project from Jonathan Harris and Sep Kamvar named as We Feel Fine [2]. This project is related to the concept of affective mapping since a web-based searching tool is used to monitor various blogs belonging to social networks over the Internet. The tool searches sentences and pictures every ten minutes that are indexed as "I feel" and "I am feeling" some sort of a personal emotional state (e.g. sad, happy, hungry, fine). The emotional states reveal the mental and physiological state of a person towards friends, relatives, and events. In We Feel Fine there is no association of human emotions to a Place, but because the usernames can be extracted from the several blogging companies (e.g. Blogger, MySpace, MSN Spaces, etc) the tool can automatically find the profile pages of the blogs' owners and retrieve their age, gender, and their location (e.g. city, state, and country).

This process is gathering between 15,000 and 20,000 emotion states per day [2], and in theory, we could determine patterns of emotions within social networks and explore their correlation among spaces (e.g. cities of different states). This example reveals an interesting methodology for gathering data about emotional states within social networks based on a collaborative process, given that those data have been voluntarily posted on the Internet, which rules out any issues of privacy in the data collection process. Additionally, it also reveals an interesting research challenge to develop more similar tools to extract data that can lead to affective mapping, with a view close to psycho-geography.

Another relevant example can be found in the artwork "Cartographies of the Strait", born in 2004 and still incomplete. The artwork emerged from a meeting of artists, activists and technological experts from both sides of the Strait of Gibraltar. Back in 2004, the aim 
was to associate the cartography of Strait of Gibraltar with the information published in Indymedia Estrecho, which is an open blog consisting of a social network of media activists [3]. Nowadays, the aim of this artwork has shifted to the visualisation of migratory, economic, political and cultural flows between the border-territory of South Europe and North Africa. In this example, the Strait of Gibraltar was a Place, since the human emotions associated to it cannot be denied. According to the Asociación Marroquí para la Comunicación, between 2,500 and 3,500 immigrants died in the course of their journey from Asia-Africa to Europe in 2008.

However, the artwork had fallen short to measure the emotions because it has primarily gathered objective data from the processes that took place in the Strait such as militarisation, migrations, displacement of capitals, communication and social movements. This points out to a major research challenge in developing approaches for gathering subjective data about human emotions that can actually prompt a dialogue between people who have different perspectives.

The last example focuses on an artwork that reminds us of the research being developed in Affective Cartography [4], which basically emphasises that "new technologies enable non-cartographers to produce viable maps, which while viewed as naïve mapping products in the eyes of cartographers, are usable products". The prototype artwork of "Much ado about nothing", conceived and being developed by the graphic design student Iván Huelves Illa, aims at the design of a new representation of the noise in a city [5]. Far from the conventional approaches that employ objective data collected in selected control points (e.g. decibel levels), the artwork seeks to gather subjective experiences from any citizen and concerned person about their perception of noise at any point of the city.

The current development of this artwork is towards a collaborative computer environment, which can expose the huge potential of citizens to become "human sensors to monitor the state of the environment" [6]. In this case, the focus is not on the presence of people and their human emotions towards a particular Place, but rather their perception of a particular phenomenon (e.g. noise) in their close-range space. It will be very interesting to follow the outcomes of this artwork and be able to test the hypothesis that the perception of high levels of noise will occur at specific Places rather than any close-range space. In other words, events can play an important role in changing the experiencing of emotional states towards a Place.

\section{Our proposed approach}

This paper advocates that the patterns obtained from the observation of different emotional states of one single Place can be useful to point up to new evidences that otherwise may probably remain unknown. Examining the implications of this view, affective mapping refers to a multidisciplinary body of research dealing with various facets of subjective experiences from individual (i.e. agent) and collective (i.e. network) points of view. The mentioned above examples support the research premise that there is always both agent and network characters of Place. In the general sense, agents in affective mapping are engaged in an on-going process of differentiation and evaluation of natural and human-made objects as well as events that coexist in a Place. This process is integral to the development of cultures, and has led to the creation of conditions and levels of adaptation that confront both positive and negative experiences. On the other hand, the examples have also revealed the reciprocities between agents' experiences of Places and the aggregation of experiential bonds between them within a network.

However, very little is know about how the patterns of collective experiences towards a Place can quantitatively as well as qualitatively be represented based on subjective data. Therefore, our research challenge is two-fold: (a) to define what Place is by pointing to ways in which emotion states can be observed and are bound together within a social network; and (b) to develop a visual representation that provides the framework for representing patterns of emotion states associated to this Place.

Towards this end, this paper will demonstrate our first results on the analysis of the correspondence obtained in the archive of Simón Ruiz that adds up to more than 50,000 documents, which were collected from a long list of sources during four decades. Remarkably, 1579 is the year with the highest rate of epistolary exchange, with 2,620 letters [7]. This commercial correspondence provides an interesting source of a personal nature abounds ("this is a sorry land for trading") that is intertwined with more objective data ("the wheat was sold at 16 ducats"). Commercial information is interspersed with opinions, memories and expectations of a personal nature that may be linked to Places, which are marked with impressions, memories, opinions and personal experiences.

In particular, we have selected 20 letters belonging to the 1560-1570 period of time to which the "bills of exchange" are currently under study at the University of Porto [8]. This objective data were not discarded, 
given that they act as markers that help us define the geographic, familiar and commercial Places where personal experiences have taken place within such a social network. The sample of 20 letters have been digitised and transcribed to modern Spanish writing [9].

Simon Ruiz was a merchant from Castile, which business expression went beyond Iberian frontiers, reaching all European trade circuits - as can be proved by the extension that his correspondence achieved all over Europe - Lisbon, Porto, Valencia, Genoa, Rouen, Rome, Venice, Lyon; and the establishment of a complex social network. His settlement in Medina del Campo made him succeed through an intensive participation in business acting as a representative to other commercial partnerships. He seems to have began the establishment of his network by placing individuals of trust in key points, as well as engaging a network of informants in important trade places, while trading textiles, but also olive oil, spices, indigo, salt or wheat, at the same time. He also began to act as a banker, exchanging foreign currency, through the use of bills of exchange, thousands of them remaining in the company archive. He became as important as some of his major clients and gave financial support to eminent personalities, as King Phillip II.

\section{The GertrudeMapper Tool}

The proposed prototype tool searches the words and accumulates their repetitions. For this reason we have decided to call it Gertrude, as a way of paying humble tribute to writer Gertrude Stein whose writing style intended to capture the immediacy of consciousness: "a visualiser is a visualiser is a visualiser is a visualiser".

As it happens with maps that portray an abstraction and synthesis of landscapes, the GertrudeMapper tool portrays an abstraction and synthesis of historical words, thus we would like to consider it as a first attempt of producing a map of historical words. Its utility derives precisely from these two qualities: abstraction and synthesis. That is because the synergy between abstraction and synthesis makes possible to reach a new insight of the universe of discourse found in the letters belonging to the social network of Simon Ruiz. In this sense, the GertrudeMapper is an exploratory tool for thought as it can lead to new understanding through vision and interaction.

The GertrudeMapper tool supports four main categories, which are: Agent, Place, Negative Emotion State, and Positive Emotion State. The category agent has been defined by the persons' names used as legal representatives. For example, a letter of exchange can involve sometimes more than eight agents. It can also refer to their location, professional and social status, honorific titles or even familiar relationships [10]. Places have been identified by keywords that report geographical, family and trade-network bonds with space, meanwhile emotion states have been identified by not just complete words such as adjectives, but also roots of words or common characters in related words; thus "avisar" ("to warn"), "aviso" ("warning"), "avisaré" ("I will warn") or "avíseme" ("warn me") were grouped as an only concept, using to this effect the group of characters "avis" as a search target. A prototype tool was designed for uploading the letters and performing an automatic reading while searching for matches with values of the aforementioned categories. This GertrudeMapper tool has also connected words between each other depending on their proximity in the examined text.

In addition, the prototype tool has been designed in order to be able to link each key word to those complete sentences in which the word can be found in its own context (more information about the prototype is found in [11]). These sentences are also interactively sent to a visual display, which can allow us to create a new, deconstructed form of reading the letters. This deconstruction is based on the link between the word that is being read and those words contained in the recent memory of the text. As a result, the link between those words that appear close to each other in the letters can be visualised as nodes and branches (Fig. 1).

Nodes represent the words in the text. Every node represents one word in the text, so that each time the prototype tool finds a new word, a new node is created, and after several runs, the tool generates aggregation patterns of nodes. The size of the node may vary and depends on the number of times the same word is read. The colour of the node is used to represent the categories to which the matching word belongs. An extra colour is reserved for those words that haven't been assigned to any category. Branches represent the relations of proximity between words in the text. They have been defined as springs that hold together the words in the letters, based on an attraction force-based algorithm [12].

The more often two words appear together, the tighter the arc that links them will be, and they closer they will get. This principle can be applied to every word in every letter; this way, each node updates its position according to the forces exerted in the whole system. 


\section{Results and Conclusions}

The preliminary results of the visualisation of the dynamic patterns of nodes and branches taking place in the system have proven to be useful to show the dynamics of how different emotion states have been bound together to Places during the $1560-1570$ period of time (Fig. 1).

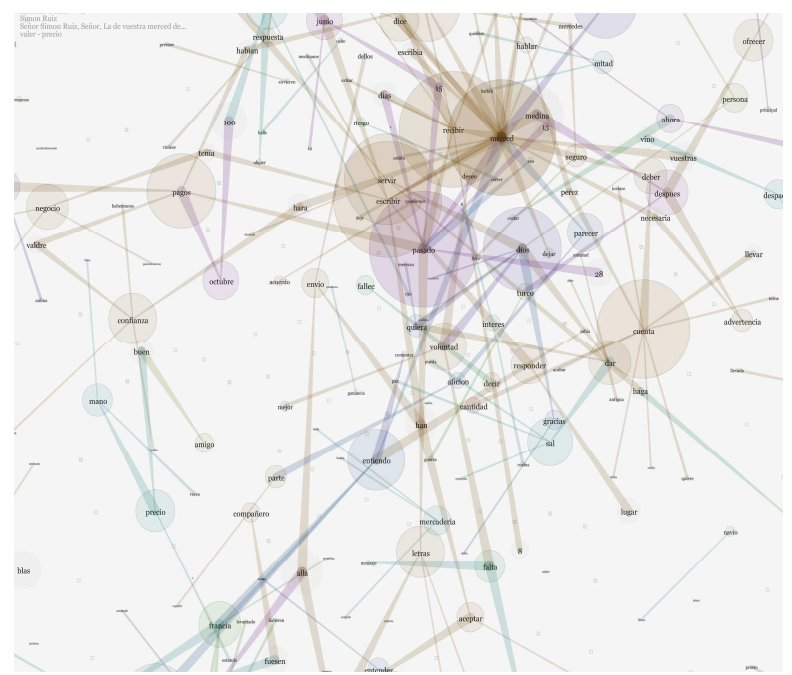

Fig. 1. The information contained in the image increases with the number of examined letters. The size of the nodes mirrors the amount of times each represented word has been mentioned in the text. The spatial distribution and size of the nodes portray the link between different words in the letters, which can be further interpreted by reading the related commercial letters.

Moreover, the preliminary results have also indicated the advantages of acquiring an early approximation of the contents of the letters based on a virtual world that allows us to glance at it and explore their contents in terms of playfulness, action, movement and interaction.

Some historians might argue that the level of uncertainty of these dynamic visual patterns is probably very high due to the subjective view of a Place, and their acceptance as a source of useful information might be cumbersome. Therefore, it is important to point out that the selection of categories and the assignment of their corresponding keywords have been carried out by the authors. It was therefore subjective and prone to errors. Nevertheless, the selected categories and keywords have been considered satisfactory to illustrate the main purpose of use of such a tool. Ideally, the GertrudeMapper tool should allow the on-the-fly compilation of such categories and keywords. This will require further developments of the
GertrudeMapper tool. For example, a future development of the tool might contemplate the elaboration of an automatic transcription of texts, or the implementation of algorithms in order to perform Fuzzy Search or approximate string matching, which is the technique of finding approximate matches to a pattern in a string [13].

The proposed GertrudeMapper tool should be seen as a way of generating new hypothesis after carrying out the affective mapping of written documents. We expect that such prototype tools will be useful to uncover facts and text information that otherwise may probably remain unknown. Finally, we would advise against approaches that rely strictly on information technology. Affective mapping implies the need for an input from other disciplines, as in our case, we have based our approach on arts, cartography, history and computer engineering. The only condition is to embed experience in the pattern of relationships that hold and unfold among people, places, and cognitive processes. Meanings and qualities of human experience that is significant for individuals or groups are then understood through reference to other forms of experience, individual and collective, past, present, and future.

\section{Acknowledgements}

This research was partially funded by the European Science Foundation under the DynCoopNet Project (Dynamic Complexity of Cooperation-Based SelfOrganising Commercial Networks in the First Global Age. We would like to thank the Portuguese team for their support and cooperation, in particular Amândio Barros, Nuno Camarinhas; João Carvalho, Joaquim Carvalho, Miguel Nogueira; Helena Osswald and Ana Ribeiro for their fruitful discussions. Finally, we would like to thank Prof. Jack Owens from Idaho State University for his innovative ideas which have motivated this research work.

\section{References}

[1] Miller, H. (2005). Social Exclusion in Space and Time. Available at http://www.geog.utah.edu/ hmiller/papers/social exclusion space time.pdf [2009, 20/05].

[2] Harris, J. \& Kamvar, S. 2008, 2008-last update, We feel fine (Project's Web Page). Available: http://www.wefeelfine.org/ [2008, 12/01]. 
[3] Indymedia Estrecho 2008, Indymedia Estrecho Project's Web Page. Available: http://estrecho.indymedia.org/ [2008, $12 / 01]$.

[4] Cartwright, W., Miles, A., Morris, B., Vaughan, L. \& Yuille, J. 2008, "Developing Concepts for an Affective Atlas" in Geospatial Vision, ed. Springer Berlin Heidelberg, pp. 219234.

[5] Huelves, I. 2008, Much Ado About Nothing. Available: http://muchadoaboutnothing.medialab-prado.es/ [2008, 12/01].

[6] Goodchild, M.F. 2007, "Citizens as sensors: the world of volunteered geography", GeoJournal, vol. 69 , no. 4, pp. 211221.

[7] Polónia, A.; Barros, A.; Nogueira, M., 2009 - Now and Then, Here and There... on business": mapping social/trade networks on First Global Age. Oral presentation at "Workshop on Mapping Different Geographies", Austria, 11 a 15 de February 2009.

[8] Polónia, A. 2008 - DynCoopNet: Portuguese research Project. Report to European Science Foundation. Available at www.dyncoopnet-pt.org/portuguese-team

and www.dyncoopnet-pt.org/about-dyncoopnet-project

[9] da Silva, J. G., 1956. Stratégie des Affaires à Lisbonne entre 1595 et 1607. Lettres marchandes des Rodrigues d'Evora et Veiga, Paris, Armand Collin

[10] Pinto, S.; and Ribeiro, A. (2009) - From Sources to GIS Results: the methodological path of the DynCoopNet Portuguese team. Paper presented at the "XVth World Economic History Congress”, Utrecht, 3-7 August.

[11] Iturrioz, T. Cano, J. and Wachowicz, M. 2010. Mapping letters through interaction design. The Cartographic Journal, accepted

[12] Holten, D. and Wijk, J.J. 2009, "Force-Directed Edge Bundling for Graph Visualization", IEEE-VGTC Symposium on Visualization, eds. H.C. Hege, I. Hotz and T. Munzner, Eurographics.

[13] Horng-Jyh, P.W., Jin-Cheon, N. and Soo-Guan, C.K. 2007, "A hybrid approach to fuzzy name search incorporating language-based and text-based principles", Journal of Information Science, vol. 33, no. 1, pp. 3-19. 